\title{
Andere Helden im populären englischen Melodrama des frühen 19. Jahrhunderts
}

\section{Christian Krug}

Auf den englischen Theaterbühnen der ersten beiden Jahrzehnte des 19. Jahrhunderts findet sich eine Reihe von Bühnenhelden, die kulturell, ethnisch oder religiös als ,Andere' markiert sind; zumeist handelt es sich bei ihnen um ,orientalische' Figuren. Solche anderskulturellen Bühnenhelden sind dem Theaterpublikum in einem hermeneutischen Sinne nicht ,fremd'; sie sind ihm im Gegenteil aufgrund ihrer Konventionalität sogar sehr vertraut. Ihre Markierung als ,Andere“ wirft daher auch nicht so sehr grundsätzliche Verstehensfragen auf - wohl aber Fragen nach Zugehörigkeiten und der eigenen Identität des Publikums. ${ }^{1}$

Bei den Repräsentationen eines anderskulturellen Heldentums im populären englischen Melodrama dieser Zeit kommen diese Fragen von Identität und Alterität in besonderer Weise zum Tragen. Dazu gehört zum Beispiel, dass ein solches Heldentum in geringerem Maße an bestimmte Figuren (etwa ,den Bühnenhelden') gebunden ist und dass heroische Attribute wie Exzeptionalität, Handlungsbereitschaft oder Transgressivität umverteilt und von Szene zu Szene unterschiedlich aktualisiert und artikuliert werden können. Warum dies so ist, möchte ich im ersten Teil meines Beitrags herausarbeiten und dabei auf spezifische Gattungskonventionen und die Bühnenpraktiken der Zeit, die soziale Verortung einzelner Theater in marginalen sozialen Räumen, aber auch auf die spezifischen Alterisierungsprozesse eingehen, die in ein solches anderskulturelles Heldentum eingeschrieben sind. Gleichzeitig möchte ich an einem Fallbeispiel, William Barrymores El Hyder; or, The Chief of the Ghaut Mountains (1818), ein analytisches Modell skizzieren, mit dem sich solche anderskulturellen Heroisierungen in Melodramen beschreiben lassen: das Modell eines dynamischen dramatischen Feldes, das sich von Szene zu Szene neu konfiguriert. In einem zweiten Teil möchte ich anhand eines weiteren Fallbeispiels, den Bühnenrepräsentationen der populären Figur Haroun Al-Rashid, exemplarisch aufzeigen, wie ein ganz bestimmtes Motiv vom , anderen Helden', die ,heroische Herrscherfigur', sich in dieser Zeit für eine Vielzahl von (ganz heterogenen) Einschreibungen und Alterisierungen öffnet.

Argumentationen anhand von Fallbeispielen sind (im positiven wie negativen Sinne) anekdotisch und hängen entscheidend von der Auswahl der Beispiele ab.

1 Zur hier verwendeten Begrifflichkeit von ,Vertrautem' und ,Fremdem' als hermeneutische Kategorien sowie des ,Eigenen' und des ,Anderen“ als Kategorien von Zugehörigkeit und Identität beziehungsweise Alterität vgl. Andrea Polaschegg: Der andere Orientalismus. Regeln deutsch-morgenländischer Imagination im 19. Jabrhundert (Quellen und Forschungen zur Literatur- und Kulturgeschichte; 35). Berlin 2005, 41-49. 
Die hier diskutierten Fallbeispiele wurden ausgewählt, da sie ein Spektrum repräsentieren: Das erste widmet sich der politisch wichtigsten Kolonie Großbritanniens, Indien, das zweite dem wohl wichtigsten Text, der populäre Vorstellungen vom orientalischen ,Anderen' in Großbritannien im 19. Jahrhundert geprägt hat den Arabian Nights' Entertainments, also den Erzäblungen aus Tausend und einer Nacht. Während im ersten Fall Fragen von anderskultureller Machtausübung systematisch ausgeblendet werden, ist der panoptische Herrscherblick Haroun Al-Rashids, der im zweiten Fallbeispiel behandelt wird, als kulturelles Phantasma ein zentrales Element von imaginären Machtbeziehungen.

Der Gegenstand meiner Überlegungen, das populäre englische Melodrama, ist dabei eine historisch und (national)kulturell sehr spezifische populärkulturelle Form. Zwar gibt es zwischen den Bühnen Frankreichs, Deutschlands und Großbritanniens einen regen Austausch von Stücken und Dramatikern; was jedoch auf den Londoner Theaterbühnen als Melodrama besucht und beschrieben wird, ist das Ergebnis von ganz eigenen institutionellen und rechtlichen Rahmenbedingungen und Diskursen, von Abgrenzungs- und Aneignungsstrategien. Wie und was Melodramen bedeuteten, ist sehr genau auf die jeweilige Gesellschaft bezogen. Dies haben sie mit dem Heroischen gemein.

Für die Analyse eines ,anderen', zumeist orientalischen Heldentums bedeutet dies, dass ein besonderes Augenmerk auf sich überlagernde Alterisierungen gelegt werden muss. Im frühen 19. Jahrhundert fungieren Melodramen (ebenso wie das sich zeitgleich etablierende Konzept des Populären) zumeist selbst bereits als ein ,Anderes': Rechtlich sind sie ein ,Anderes' der sogenannten legitimen Dramenformen Komödie und Tragödie, die bis 1843 in London einzig an den drei mit königlichem Patent ausgestatteten (,lizenzierten') Theatern aufgeführt werden durften. ${ }^{2}$ Topographisch siedeln sie sich zunächst an anderen städtischen Orten, etwa dem ärmeren East End Londons, an, dessen Theaterpublikum zuweilen als soziales ,Anderes' gefürchtet wird. Schließlich erscheint das Affektive des Melodramas den bürgerlichen Mittelschichten als ästhetisch ,Anderes'. Für die Analyse ,anderer Helden ist daher eine erste Prämisse, dass sich intrakulturelle und interkulturelle Prozesse des Othering immer schon überlagern.

Weitere methodologische Konsequenzen ergeben sich aus der Struktur des Melodramas und seiner Aufführungspraxis. So sind die dramatischen Strukturen gerade nicht durch Kohärenz, sondern durch sehr heterogene Momente („situation and incident $\left.{ }^{\prime \prime}\right)^{3}$ geprägt. Melodramen etablieren zudem ganz eigene emotionale Regime (die beispielsweise szenisch-tonal geprägt sind) ${ }^{4}$ und produzieren eine

2 Vgl. Jane Moody: Illegitimate Theatre in London, 1770-1840. Cambridge 2000.

3 Vgl. Johann N. Schmidt: Ästhetik des Melodramas. Studien zu einem Genre des populären Theaters im England des 19. Jahrhunderts. Heidelberg 1986, 200.

4 Vgl. Conrad Joy Bishop: Melodramatic Acting. Concept and Technique in the Performance of Early Nineteenth Century English Melodrama. Diss., Stanford University 1967, 51-52. 
Affekt-Intensität, die danach drängt, in Zeichenhaftigkeit überführt zu werden. ${ }^{5}$ Dadurch verkomplizieren sie konventionelle heuristische Unterscheidungen zum Beispiel von Emotion (im Sinne William Reddys als soziale Kommunikationsform, die sich in - auch konkurrierenden - historischen Regimen anordnet $)^{6}$ und Affekt (als vermeintlich vorindividuelle Intensität). Auch sind (Helden-)Figuren allenfalls innerhalb ganz allgemeiner Paradigmen im Dramenverlauf stabil, ansonsten jedoch gerade von relationaler Bezüglichkeit. Zeitgenössische Diskurse über das Melodrama weisen auf andere Logiken von Figuren- und Handlungskonstruktion und -rezeption hin, ${ }^{7}$ innerhalb derer sich Helden im konventionellen Sinne nur schwer fassen lassen. Für die Analyse erfordert dies entsprechend eine Perspektive, die auf Momente fokussiert und variable, instabile Heroisierungsverfahren im Blick behält. ${ }^{8}$ Dies wird bereits in der vielbeschworenen Krise des melodramatischen Bühnenhelden evident, die in der Forschungsliteratur vielfach beschrieben, aber selten überzeugend erklärt wurde. Traditionelle (männliche) Bühnenhelden hatten keinen leichten Stand in den englischen Melodramen des frühen 19. Jahrhunderts. Als bühnenwirksamer galt die Rolle des Schurken, emotional und affektiv aufgeladener zumeist die der Heldin, und im Spannungsfeld zwischen diesen beiden Figuren droht der melodramatische Bühnenheld dramaturgisch fast überflüssig zu werden. In seiner klassischen Studie zum englischen Melodrama charakterisiert Michael Booth ihn entsprechend als konventionell passiv, schwach und „rather stupid“. ${ }^{9}$ Dass auch in der Forschungsliteratur zum Melodrama vergleichsweise selten auf männliche Heldenfiguren fokussiert wird, ist jedoch nicht nur symptomatisch für eine solche veritable Krise des Bühnenhelden. Es mag auch Ausdruck einer analytischen Krise sein, die sich zum einen daraus ergibt, dass im Melodrama Repräsentationen von Heldentum in sich häufig widersprüchlich sind, ${ }^{10}$ und zum

5 Vgl. hierzu die klassische Studie von Peter Brooks: The Melodramatic Imagination. Balzac, Henry James, Melodrama, and the Mode of Excess. New Haven und London ${ }^{2} 1995$.

6 William Reddy: The Navigation of Feeling. A Framework for the History of Emotions. Cambridge 2001.

7 Eine gute Rekonstruktion zeitgenössischer Diskurse liefert der immer noch einschlägige Aufsatz von Kurt Tetzeli von Rosador: Victorian Theories of Melodrama. In: Anglia 95, 1977, 87-114.

8 Die Analyse von El Hyder wird zeigen, dass allzu homogenisierenden Bedeutungszuschreibungen, wie sie Melodramen selbst zum Beispiel durch End-Tableaus suggerieren, durchaus misstraut werden kann: Die letzten Szenen von Melodramen etablieren häufig retroaktiv eine vermeintliche Stabilität von Figuren und Werten, die sich in der Theatererfahrung zuvor nicht unbedingt gefunden haben muss.

9 Michael R. Booth: English Melodrama. London 1965, 17, 18.

10 Jacqueline Bratton argumentiert, dass Repräsentationen von Heldentum immer wieder von anti-heroischen Momenten durchkreuzt werden: „melodramatic British heroism has vital anti-heroic dimensions. [...] It is both tied to narrow stereotypes, and [at the same time] as inclusive as possible, in class and regional/national terms; it admits non-heroic figures as heroes, and [it admits] open statements of possibly subversive views and feelings" (Jacqueline S. Bratton: British Heroism and the Structure of Melodrama. In: Jacqueline S. Bratton [et al.] (Hg.): Acts of Supremacy. The British Empire and the Stage, 1790-1930. Manchester 1991, 18-61, hier 27). 
anderen daraus, dass sich seinen relational bezüglichen Figuren bei genauerer Betrachtung dann doch nicht scherenschnittartig ideologische Funktionen zuordnen lassen. Anhand von Barrymores El Hyder möchte ich mich deshalb dem Bühnenhelden analytisch anders nähern und dessen ,Krise in den Kontext instabiler Heroisierungsverfahren setzen.

\section{Die Zirkulation heroischer Energien in William Barrymores El Hyder}

In diesem Melodrama kämpft der charismatische Stammesführer El Hyder darum, einen jungen Prinzen aus der Gefangenschaft des tyrannischen Radschas Hamet zu befreien; dieser hält den Prinzen und dessen Mutter, um deren Hand er wirbt, in einem Harem gefangen. El Hyder gelingt es schließlich mit seinen Truppen, Hamet zu bezwingen und den rechtmäßigen Thronanspruch des Prinzen durchzusetzen. Die Titelfigur dieses Melodramas basiert auf einem indischen Feldherrn, Haidar Ali (1721-1782), ${ }^{11}$ der die Truppen der britischen East India Company in den ersten beiden Mysore-Kriegen (1767-1784) besiegte und für die britische Öffentlichkeit einen der bekanntesten Gegner der Briten in Indien darstellte. ${ }^{12}$ An seiner Figur lassen sich die Heroisierungspotenziale eines , anderen' Helden gut demonstrieren. Die Analyse zeigt aber auch, dass heroische Attribute in einem Melodrama über unterschiedliche Figuren (,eigene` wie ,andere) verteilt und zum Teil je nach Szene dynamisch neu konfiguriert werden - und dass lokale Kontexte, bis hin zum sozialen Umfeld einzelner Theater, solche spezifischen Heroisierungen bedingen.

Auf den ersten Blick scheint Hyder Ali ein ungewöhnlicher Held in einem Londoner Theater zu sein, und ein indischer Befreiungskampf gegen eine tyrannische Herrschaft ein ungewöhnliches Sujet. Die East India Company hatte in einer ganzen Reihe von Publikationen, etwa in captivity narratives über britische Gefangene, systematisch versucht, den historischen Haidar Ali zum Gegner nationaler britischer Interessen zu machen. ${ }^{13}$ Dennoch wird er 1818 im Süden und Osten Londons zu einem local hero, dessen Heroisierung eng an die spezifischen Bühnen geknüpft ist, auf denen er aufgeführt wurde. Dies war erstens das neu gegründete Royal Coburg Theater südlich der Themse (das heutige Old Vic). Das Coburg gab sich eine ,radikale‘ Ausrichtung (es positionierte sich zum Beispiel politisch klar gegen den Prinzregenten, den späteren König Georg IV.), und sein Publikum bestand

11 Narendra Krishna Sinha: Haidar Ali. Calcutta ${ }^{3} 1959$.

12 Vgl. Rajat Kanta Ray: Indian Society and the Establishment of British Supremacy, 17651818. In: P. J. Marshall und Alaine Low (Hg.): The Oxford History of the British Empire. Bd. 2: The Eighteenth Century. Oxford 1998, 510-520.

13 Vgl. Linda Colley: Captives. Britain, Empire and the World 1600-1850. London 2003. David Worrall: Harlequin Empire. Race, Ethnicity and the Drama of the Popular Enlightenment (The Enlightenment World; 1). London [u.a.] 2007, 82. 
zu einem hohen Anteil aus Dienerschaft und Arbeitern. ${ }^{14}$ Drei Jahre später wechselte das Melodrama in das etwa 1600 Personen fassende Royalty Theatre im Stadtteil Tower Hamlets; dieses lag in unmittelbarer Nachbarschaft zum neu errichteten East India Dock und rekrutierte sein Publikum auch aus den multi-ethnischen Hafenarbeitern der East India Company. ${ }^{15}$ Dass das Melodrama mit seinem orientalischen sowie muslimischen Helden in diesen Theatern Erfolg hatte, ist vielleicht auch ein Indiz dafür, dass sich die von Edward Said beschriebenen ,klassischen' orientalistischen Diskurse eher in sanktionierten kulturellen Bereichen, etwa den offiziellen Publikationen der Company, aber nicht notwendigerweise im Bereich einer lokal umgrenzten Populärkultur durchsetzen konnten. ${ }^{16}$

Der Bühnenheld El Hyder erweist sich direkt zu Beginn der ersten Szene als charismatischer Anführer von patriotischen indischen Truppen. El Hyder ist dabei der Fokus der „Patrioten“; $; 1$ in ihm verdichtet sich ihr Kampf - und dies im Wortsinne, denn die dramatische Ausgestaltung der gerade gekämpften Schlacht erfolgt als innerer Nachvollzug:

EL HYDER Yes, Moloc, even so. But why these downcast eyes? Am I to read mistrust? El Hyder doubted! Did he not perform his duty?-Did he not protect his charge as long as man was man? Behold! are these not witnesses? Will not these wounds, still reeking with my blood convey conviction to your minds? Mark! here stood the tent that held my sacred charge,-far beyond our flank, stretched the enemy's horse-our wing was broken, over-whelmed, discomfitted-the Princess viewed it, and cried for HyderHyder flew to them-we were surrounded-foe on foe! I still maintained my ground, but they seized the Princess and her son-I heard their cries-saw them borne awaywhen I, with a Boa's rage, rushed to the rescue-a conquering arm struck me downand Hyder saw no more!

MOLOC Oh, Hyder, do not think we doubt you! Quick, comrades, from his mind dispel such thoughts-our country's love, our country's hope is-

OMNES Hyder! ${ }^{18}$

14 Vgl. Frederick Burwick: Playing to the Crowd: London Popular Theatre, 1780-1830. New York 2011, 194-200. Worrall: Harlequin Empire (Anm. 13), 100-101. Moody: Illegitimate Theatre (Anm. 2), 167-168.

15 Worrall: Harlequin Empire (Anm. 13), 101. David Worrall hat die Aufführungen von Barrymores Melodrama umfassend rekonstruiert, vgl. ebd., 81-105 sowie die bis auf wenige Änderungen wortgleiche Diskussion in David Worrall: Islam on the Romantic Period Stage. Hyder Ali, Tippoo Saib and Beyond the Captivity Narrative. In: Michael T. Davis und Paul A. Pickering (Hg.): Unrespectable Radicals? Popular Politics in the Age of Reform. Aldershot [u.a.] 2008, 167-184.

16 Vgl. Edward Said: Orientalism. Western Conceptions of the Orient. London 1978. Worrall: Harlequin Empire (Anm. 13), 81-87.

17 William Barrymore: El Hyder, the Chief of the Ghaut Mountains: A Grand Eastern Melo-Dramatic Spectacle. In Trwo Acts (T. H. Lacy). London [o. J.], 25. Historisch wurden die Marathen, die El Hyder in diesem Drama anführt, 1803 und 1818 (nur wenige Monate vor der Erstaufführung) von den britischen Truppen besiegt, vgl. Ray: Indian Society (Anm. 12), 511, 522.

Barrymore: El Hyder (Anm. 17), 4. 
Hyder Alis Heroisierung erfolgt hier in Form eines Stücks im Stück, einer metatheatralen Miniatur. Die abschließende Akklamation durch die „patriotischen Stammesfürsten "19 bestätigt nicht nur Alis Selbstzuschreibung von Heldentum, sondern markiert auch, entsprechend der melodramatischen Struktur von situation and incident, das Ende dieser ersten ,Aufführung' in einer überaus heterogenen Szenenfolge. Kaum hat nämlich das Drama sein Figurenpersonal etabliert und seine Themen besetzt, zieht es seinen Helden sofort wieder aus dem Verkehr. Es belegt ihn in seiner nächsten Szene de facto mit einem inneren, moralischen Handlungsverbot sowie einer vollständigen sozialen Isolation, die sich unter dem konstanten Blick der Gemeinschaft vollzieht: Hyder Ali, der Fluchtpunkt des Kampfes der Patrioten, darf sich in ihrer Gemeinschaft frei bewegen, jedoch keinerlei soziale Kontakte aufnehmen. ${ }^{20}$

Dramaturgisch gesprochen gibt es damit einen Stau an heroischer Energie. Deren Überschuss sucht sich von Szene zu Szene neue Ventile und überschreitet dabei sowohl geschlechtliche als auch ethnische und nationale Grenzen: Spektakuläre Kämpfe mit Dolchen und Pistolen führt zum Beispiel eine indische Prinzessin. Zwei britische Seemänner, die an der Küste von Delhi gestrandet sind (und dies im südindischen Mysore), stellen wiederum einen anderen konventionellen Heldentyp bereit, die Bühnenfigur des Jack Tar ${ }^{6}$ - einen britischen Matrosen mit einem Herz aus Gold, der für Gerechtigkeit und Großbritannien kämpft. Dass sich in Melodramen häufig Seemänner aufs Land verirren, ist übrigens nur vordergründig inkongruent. Es ist vielmehr symptomatisch: Um 1820 waren Soldaten äußerst ambivalent konnotiert; zum einen galt das Heer traditionell als Auffangbecken für Kriminelle und Arme, zum anderen ließen sich einige der Soldaten, die aus den französischen Revolutionskriegen zurückehrt waren, nur schwer wieder in die Gesellschaft integrieren. Da militärischer Ruhm im kulturellen Gedächtnis eng mit den Erfolgen der britischen Navy verknüpft war, fungierte zumeist der Seemann als prototypischer militärischer Held. ${ }^{21}$

Der erste der Seemänner in El Hyder, Mat Mizen, appelliert dabei in einem beständigen Wortschwall an vermeintlich geteilte nationale Eigenschaften und Werte. Seine Funktion ist die Anbindung an eine imaginierte nationale Gemeinschaft; er produziert clap traps, das heißt, er appelliert in Sentenzen und lässt diese über den eingeholten Beifall des Publikums bestätigen - ein solches gemeinsames Klatschen stiftet in der Praxis aber auch eine viel enger umgrenzte, lokale Gemeinschaft in

19 Ebd., [2].

20

Anders argumentiert Jane Moody, die vor allem auf die Transgressivität der Figur abhebt: „despite his moral heroism, the sailor remains an unknowable stranger on the very edge of modern civilisation. [...] In the wake of mass post-war demobilisation, the brave, brusque but unpredictable sailor had usurped the [role of the] wild man as a theatrical subject precisely because he epitomised contemporary fears about the identity of the civil and the monstrous in late Georgian society“ (Moody: Illegitimate Theatre [Anm. 2], 97-98). 
den einzelnen Theatern. ${ }^{22}$ Der zweite Seemann, Harry Clifton, übernimmt eine leicht andere Funktion und verkörpert vor allem das kindlich-unschuldige Heldentum der frühen Jack Tar-Figur. Er lässt sich (ähnlich der Figur Harpo bei den Marx Brothers) als Verkörperung des Freudschen Es beschreiben; mit kindlich-naiver Freude agiert er seine Triebe aus und stellt dem Drama dadurch Momente sozialer und sexueller Transgressivität bereit. ${ }^{23}$ Es heißt oft, dass Melodramen mit dieser Figur des Jack Tar der Repräsentation eines, eigenen' Helden am nächsten kommen. Tatsächlich funktioniert aber auch diese Figur überhaupt nur in der ,Fremde', in ,xenotopen' Räumen. ${ }^{24}$ Sobald sie nämlich in heimische, englische Kontexte gesetzt wird - so etwa im domestic melodrama ab den 1830er Jahren - wird sie aufgrund ihrer Transgressivität zum sozialen Störfall. Bei dem zweiten britischen Seemann resultieren Infantilisierung, Transgressivität und Exzess dabei nur teilweise in einer Exotisierung der Figur, wie sie ansonsten gattungstypisch betrieben wird. Lokal wird ihr Othering anders, nämlich geschlechtlich, betrieben: Die Figur wurde 1818 am Coburg von „Mrs. W. Barrymore“ gespielt, der Ehefrau des Autors und Schauspielers, der den Tyrannen gab. ${ }^{25}$ In Melodramen ist ein solches geschlechtliches Othering insofern möglich, als es als ,Mischgattung' traditionell eine Nähe zur Pantomime aufweist, in welcher die Rolle des Principal Boy immer von einer Frau gespielt wird. ${ }^{26}$

22 Vgl. hierzu auch Bratton: British Heroism (Anm. 10), 23. Auf den nicht-lizenzierten Bühnen (wie dem Coburg) können solche Momente imaginierter Gemeinschaften noch zusätzlich lokal geprägt sein, da lokale Schauspiel-Stars teilweise während einer Aufführung aus ihrer Rolle traten, um (vergleichbar mit dem modernen stand up) lokale Ereignisse zu kommentieren. Aufführungen werden von solchen isolierten, tendenziell handlungs- und kontextfreien Momenten ,punktiert - auch in diesem Sinne stellen Melodramen kein homogenes oder lineares Theatererlebnis bereit.

23 Entwürfe einer Genealogie der Tar-Figur finden sich in Bratton: British Heroism (Anm. 10), 43, und in Heidi J. Holder: Melodrama, Realism and Empire on the British Stage. In: Jacqueline S. Bratton [et al.] (Hg.): Acts of Supremacy. The British Empire and the Stage, 17901930, Manchester 1991, 129-149. Demnach habe sich diese Figur von einem kindlichen und vermeintlich unschuldigen Heldentum in den ersten zwei Jahrzehnten des 19. Jahrhunderts hin zu einer abgeklärteren und sentimentalisierten Form ab den 1830er Jahren entwickelt. Zu Ende des Jahrhunderts werde ein nunmehr nostalgisch verklärter Jack Tar zunehmend sozial instrumentalisiert.

24 In Anlehnung an Michel Foucaults Konzept der Heterotopie sind ,Xenotopien' als Räume beschrieben worden, ,innerhalb derer Fremdheit hergestellt und inszenatorisch vermittelt wird. Ihre primäre Funktion im Europa des 19. Jahrhunderts besteht darin, einem heimischen Publikum, für das der Umgang mit dem äußeren und vor allem dem exotischen Fremden keineswegs alltäglich ist, strukturierte und räumlich erfahrbare Alteritätsserfahrungen zu ermöglichen. Folgerichtig zielen Xenotopien auf ein breites, gegen Ende des Jahrhunderts sogar auf ein Massenpublikum ab und tragen so zur Popularisierung des Fremden bei." (Volker Barth [et al.]: Einleitung. In: Volker Barth [et al.] (Hg.): Xenotopien. Verortungen des Fremden im 19. Jahrhundert (Kulturgeschichtliche Perspektiven; 9). Münster [u.a.] 2007, 7-23, hier 15-16).

25 Barrymore: El Hyder (Anm. 17), [2]. Vgl. Worrall: Harlequin Empire (Anm. 13), 84.

26 Vgl. Karl Sabbagh: The Arabian Nights in British Pantomime. In: Philip F. Kennedy und Marina Warner (Hg.): Scheherazade's Children. Global Encounters with the Arabian Nights. New York 2013, 265-273, hier 267. 
In der Schlussszene von El Hyder stehen die beiden Briten allerdings ebenfalls nicht mehr im Zentrum eines heroischen Handelns. Ihre Anzahl hat sich nämlich in der vorletzten Szene vervielfacht - eine größere Gruppe weiterer britischer Seemänner muss ebenfalls das Landesinnere durchzogen und dabei auch schwere Artillerie mit sich geführt haben. Aufgrund der schieren Anzahl der britischen ,Seemänner in El Hyder entspricht deren Handeln allerdings nicht mehr den konventionellen ideologischen Repräsentationen von Heldentum: Im frühen kolonialen Melodrama wird die britische Militärmacht systematisch verdeckt, individualisiert und über wenige Einzelfiguren repräsentiert - meist die gallant ferw, die sich gegen eine Übermacht durchsetzen. ${ }^{27}$ So ergibt sich der Bedarf für eine letzte Verschiebung von heroischer Energie: Ein Kampf der gallant ferw war ja genau das Thema der ersten Szene um El Hyder, den durch ein inneres Handlungsverbot ruhiggestellten Titelhelden. So schließt sich der dramaturgische Kreis des Dramas, das nun zu seinem ersten Heldennarrativ zurückkehrt und den indisch-muslimischen Held reaktiviert. Szenische und emotionale Höhepunkte sind entsprechend der Kampf der Prinzessin mit ihrem Haremswächter und schließlich El Hyders Kampf mit dem „Tyrannen“ Hamet und seinen Männern. ${ }^{28}$

Als Beschreibungsmodell für Melodramen wie dieses bietet sich das eines dynamischen Feldes an, in dem heroische Energie gewissermaßen zirkuliert und dann, gemäß der melodramatischen Struktur von situation and incident, in ihren verschiedenen Aspekten von unterschiedlichen Figuren ausagiert wird - ,eigenen ebenso wie ,anderen'. ${ }^{6}$ Der Titelheld bleibt dabei ihr Fluchtpunkt. Was das Melodrama jedoch nicht wagt, ist, ihm nach gewonnenem Kampf politische Macht zu übertragen (die der historische Haidar Ali sehr wohl hatte). Der Bühnen-Hyder kämpft auch gar nicht für sich, sondern für ein junges Prinzenkind, so dass sich die Frage von Herrschaft gar nicht stellen muss. ${ }^{30}$ Stattdessen imaginiert das Drama über die Figur des Hamet symbolisch, was passieren könnte, wenn ein anderskultureller Held wie El Hyder selbst Herrschaft erlangen würde. Hamet wird vom Star

Barrymore: El Hyder (Anm. 17), 4 und passim. Auch das schlussendliche Schwenken der britischen Fahne lässt sich nicht ausschließlich auf nationalistische Bedeutungen verengen (vgl. auch Anm. 8); es markiert vielmehr die im Drama immer wieder beschworene ,Freiheit', die längst mit ,indischer Befreiung' überlagert wurde und nun zwischen ,eigenen ${ }^{6}$ (beziehungsweise je nach Publikum, anderen') Sinneinschreibungen oszilliert.

29 In seinen dynamischen Heroisierungen ist das Drama damit deutlich komplexer, als es seine Zusammenfassungen in kritischen Studien vermuten lassen. Hier dient es zumeist als prototypisches Beispiel ,des' kolonialen britischen Theaters. „Here, and in similar plays, spectators saw Britain's imperial ambitions dramatized as an heroic crusade for liberty against usurping tyrants and barbaric native customs" (Jane Moody: The Theatrical Revolution, 1776-1843. In: James Donohue (Hg.): The Cambridge History of British Theatre. Bd. 2: 1660 to 1895. Cambridge 2004, 199-215, hier 205).

30 Worralls Interpretation, Barrymores El Hyder repräsentiere einen islamischen ,Herrscher ${ }^{6}$ als heroische Figur - „who affirmed [his] sovereignty and delivered rousing speeches to [his]

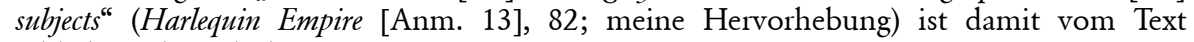
schlicht nicht gedeckt. 
des Ensembles gespielt und fungiert als El Hyders alter ego - als gefallener Heros, der sich in den politischen Zwängen der Macht verstrickt hat. Auch hier sind die sich überlagernden Alterisierungen spannend: Auf den Bühnen des Coburg und des Royalty Theatre, beide sogenannte minor theatre ohne königliches Patent, wird dieser „Tyrann“ durch seine deutlichen Bezüge zur Shakespeareschen Tragödie, insbesondere Richard III, als ,Anderer' markiert. Die Differenzlinien, die zwischen den komplementären Figuren eingezogen sind, verlaufen dabei nicht nur zwischen sehr unterschiedlich konnotierten Gattungen, Tragödie und Melodrama, sondern zwischen einer „legitimen“ und „illegitimen Theaterkultur“31 mit ihren jeweiligen sozialen Orten. Solche Linien sind allerdings fließend: Shakespeare selbst war erst ab Mitte des 18. Jahrhunderts kulturell nobilitiert und zu Beginn des 19. Jahrhunderts im Zuge der Herausbildung einer ästhetisierten Hoch- beziehungsweise Elitenkultur gewissermaßen entpopularisiert worden. Kurz nach den hier behandelten Aufführungen versuchen einige minor theatres dann verstärkt, sich Shakespeare als ,Eigenes' anzueignen, um dadurch ihr kulturelles Prestige zu steigern: Sie werben öffentlich mit ihrer Verortung südlich der Themse (Shakespeares ersten Spielorten) und engagieren namhafte Shakespearedarsteller für Gastspiele; das Coburg zum Beispiel organisiert 1819 ein Gastspiel von Junius Brutus Booth als Richard III. oder 1831 von Edmund Kean (der jedoch gegen den local hero des Theaters, den ,Volksschauspieler' Cobham, nicht bestehen kann). ${ }^{32}$ Das Othering des indisch-muslimischen El Hyder über die Dramenfigur des nationalisierten, angeblich hochkulturellen Shakespeare muss damit in sich widersprüchlich bleiben. ${ }^{33}$

\section{Der andere Held als kulturelles Phantasma}

In den populären Melodramen der ersten beiden Jahrzehnte des 19. Jahrhunderts werden Heroisierungen anderskultureller Figuren zumeist durch Mechanismen der Ab- und Ausgrenzung flankiert, und wenn wie in Barrymores El Hyder das heroische Handeln eines anderen Helden in der Möglichkeit einer herrschaftlichen Machtausübung resultieren könnte, dann wird diese meist systematisch ausgeblendet. In meinem zweiten Fallbeispiel steht dieser Aspekt der Herrschaft eines anderskulturellen Heldens nun im Mittelpunkt. Es handelt sich um die Anfang des 19. Jahrhunderts wohl populärste Figur der zahlreichen Bühnenadaptationen der Arabian Nights' Entertainments, den in Verkleidung durch Bagdad ziehenden

31 Moody: Illegitimate Theatre (Anm. 2), 10.

32 Vgl. ebd., Kap. 4, insbesondere 136-137, sowie Jane Moody: Romantic Shakespeare. In: Stanley Wells und Sarah Stanton (Hg.): The Cambridge Companion to Shakespeare on Stage. Cambridge 2002, 37-57.

33 Ohnehin überlagern sich Kanonisierungs- und Popularisierungsbestrebungen und stellen im frühen 19. Jahrhundert gerade keine klaren Dichotomien dar, vgl. Doris Feldmann und Christian Krug: Zur Geschichte des Populären. Konzepte und Praktiken englischer Populärkultur im 19. Jahrhundert. In: Sabine Friedrich und Dirk Niefanger (Hg.): Populärkultur. Würzburg [erscheint 2016]. 
Kalifen Haroun Al-Rashid. Seine Herrschaft ist zu Beginn der Dramen bereits etabliert; es stellt sich damit nicht mehr die Frage, ob sie gewonnen werden darf, sondern wie sie ideologisch legitimiert werden kann, und was die Popularität der Figur auf den englischen Theaterbühnen des frühen 19. Jahrhunderts begründet. Dabei möchte ich mich vor allem einem bestimmenden Element widmen: der Inszenierung des machtvollen, gesellschaftliche Identität stiftenden Blicks dieser heroischen Herrscherfigur.

Das Motiv des verkleideten Herrschers selbst hat eine lange Geschichte im englischen Theater. $\mathrm{Zu}$ bestimmten Zeiten (insbesondere Anfang des 17. Jahrhunderts) findet es sich gehäuft, ${ }^{34}$ und auch zu Beginn des 19. Jahrhunderts haben verkleidete Herrscher Konjunktur - nicht nur auf den Theaterbühnen, sondern auch in populären politischen Diskursen der Zeit. ${ }^{35}$ In den Dramen um Haroun stehen dabei meist Inszenierungen seines omnipräsenten Blicks im Mittelpunkt. Dieser Blick, der immer alles sieht, wird nicht nur in spektakulären Bühnenmomenten inszeniert, er durchwirkt die Dramen auch in vielen kleinen Momenten. Dabei machen sich Dramatiker auch die spezifische Aufführungspraxis von Melodramen zunutze. Da Schauspieler vorwiegend in einer Linie auf der Bühne agieren und dabei meist noch dem Publikum frontal zugewandt sind, existieren Bühnenorte oberhalb dieser Ebene (up stage); diese extra-diegetischen Bühnenorte bleiben für das Publikum sichtbar, werden von den handelnden Figuren aber ignoriert. ${ }^{36}$ Der Kalif kann dort verortet werden, so dass sein Herrscherblick gewissermaßen ,phantasmatisch ${ }^{6}$ - über der Szene schwebt. ${ }^{37}$

Durch solche (und viele weitere) Inszenierungsformen stellt der Herrscherblick einem Londoner Theaterpublikum ein kulturelles Phantasma bereit, über das die Herrschaft eines anderskulturellen Helden imaginiert werden kann. Alain Grosrichard hat die Funktion dieses Phantasmas 1979, fast zeitgleich zu Edward Said, in der ,anderen' Studie zum französischen Orientalismus sehr präzise gefasst. ${ }^{38} \mathrm{Er}$ beschreibt das Phantasma des osmanischen Despotismus als eine im Grunde me-

34 Vgl. bereits Victor Oscar Freeburg: Disguise Plots in Elizabethan Drama. A Study in Stage Tradition. New York 1915. Leonard Tennenhouse: Power on Display. The Politics of Shakespeare's Genres. London ${ }^{2} 2005$. Kevin A. Quarmby: The Disguised Ruler in Shakespeare and his Contemporaries. London [u.a.] 2012.

35 Vgl. Linda Colley: The Apotheosis of George III. Loyalty, Royalty, and the British Nation 1760-1820. In: Past and Present 102, 1984, 94-129.

36 Vgl. Bishop: Melodramatic Acting (Anm. 4), 200-204.

37 Ein gutes Beispiel hierfür findet sich in Thomas Dibdin: Il Bondocani, or, The Caliph Robber: A Comic Opera, in Three Acts. London 1801 [Uraufführung 1800], 28.

38 Alain Grosrichard: The Sultan's Court. European Fantasies of the East, übers. von Liz Heron. London [u.a.] 1998 [Französische Erstausgabe 1979]. Vgl. auch Mladen Dolar: Introduction. The Subject Supposed to Enjoy. In: Alain Grosrichard: The Sultan's Court. European Fantasies of the East, übers. von Liz Heron. London [u.a.] 1998, ix-xxvii. Im Gegensatz zu Saids Orientalism-Studie ist The Sultan's Court stark von Lacan beeinflusst und hat einen deutlich begrenzteren Fokus; Grosrichard widmet sich ausschließlich dem kulturellen Phantasma des „asiatischen Despotismus“ (x) in der französischen Literatur des 18. Jahrhunderts. 
chanische Machtapparatur, die vor allem auf zwei Elementen basiert: dem omnipräsenten Blick und dem Namen des Despoten, der als machtvoller Signifikant durch das Reich zirkuliert. ${ }^{39}$ Für Grosrichard fungiert das Phantasma als das ,Andere` einer aufgeklärten, rationalen französischen Gesellschaft; es bildet ihren ,realen Kern' und liefert Subjekten eine imaginäre Begründung dafür, warum sie sich Macht unterordnen (und unterordnen wollen), noch bevor sie dann ihre Stellung zur Macht - ganz , aufgeklärt' - rationalisieren. ${ }^{40}$

Henry Milners Barmecide (ebenfalls 1818) inszeniert Haroun als einen solchen benevolenten ,Despoten'. Despotismus ist dabei eine Machtform, die neben dem öffentlichen und politischen Bereich dezidiert auch das Häusliche und den Bereich der Familie umfasst; etymologisch bedeutet ,Despot ${ }^{\star}$ auch Hausherr, und für Grosrichard beziehen Repräsentationen von Despotismus ihre Metaphern daher folgerichtig auch aus Familienbeziehungen ${ }^{41}$ (etwa als „der Vater des Volkes“, eine zentrale Bezeichnung Haroun Al-Rashids in den hier behandelten Melodramen). Haroun muss in Barmecide lernen, kein „Tyrann“ zu sein, ${ }^{42}$ darf dafür jedoch als liebenswerter Despot weiter regieren. In Bezug auf die melodramatischen Heroisierungen Harouns wäre damit eine erste These, dass seine Figur es dem Publikum ermöglicht, die eigene Anbindung an Macht genussvoll zu imaginieren, während es gleichzeitig auf einer symbolischen Ebene aktuelle sozio-politische Fragen verhandelt. Unabhängig vom Ausgang dieser Verhandlungen hat die Anbindung an Macht jedoch längst stattgefunden. Haroun, so ließe sich überspitzt formulieren, ist der anderskulturelle Held einer perfiden Selbstunterwerfungsfantasie.

In den Melodramen funktioniert dieses Prinzip zum Beispiel so, dass Haroun die Funktion eines ,panoptischen Übervaters' übernimmt. Er erlaubt es einem städtischen Theaterpublikum dadurch, sich verloren geglaubten, paternalistischfeudalen Gesellschaftsstrukturen in einer nostalgisch verklärten, exotisierten Form noch einmal zu vergewissern. ${ }^{43}$ Insofern ermöglicht der anderskulturelle Held auch eine Anbindung an die eigene imaginierte Vergangenheit. So gerahmt können die Melodramen dann eine ganze Fülle von sehr spezifischen aktuellen sozialen Fragen diskutieren. ${ }^{44}$ Dazu gehören zum Beispiel Arbeit und Armut: Armut ist eine

39 Grosrichard: The Sultan's Court (Anm. 38).

40 Vgl. auch Dolar: Introduction (Anm. 38), xv-xvi.

41 Grosrichard: The Sultan's Court (Anm. 38), 3-16.

42 Henry Milner: Barmecide; or, The Fatal Offspring. A Dramatic Romance. London 1818, 36. Giafar erklärt dem Kalifen: „Your only zealous friend and defender your tyranny has driven from your cause, and made your enemy. For this you are indebted to your ungoverned passion. Justice and mercy constitute a sovereign's brightest glory. Had you listened to their voice, you had not been thus disgraced." (51)

43 Vgl. zu diesem und den folgenden Punkten ausführlicher Christian Krug: Das Eigene im Fremden. Orientalismen im englischen Melodrama, 1790-1840 (Schriftenreihe Literaturwissenschaft; 53). Trier 2001, 139-148.

44 Dazu gehört es auch, eine empfundene Kluft zwischen Rechtsempfinden und Gesetz oder zwischen dem Staat als Idee und seinen real existierenden Verwaltungsinstitutionen zu überbrücken (so 1800 in Dibdin: Il Bondocani [Anm. 37]). 
soziale Kategorie, die im frühen 19. Jahrhundert immer feiner ausdifferenziert wurde. ${ }^{45}$ In Thomas Dibdins Azim; or Wants and Superfluities (1818) wird diese Differenzierung symbolisch durchgespielt. Haroun führt ein Sozialexperiment mit seinem armen Titelhelden durch, in dem dieser unter ständiger, verdeckter Beobachtung Bedarfsartikel und Luxusgüter unterscheiden und kategorisieren muss. (Das ultimative Luxusgut, das Azim nicht verlangen darf, ist - natürlich - der unverstellte Blick. Dieser bleibt dem Kalifen vorbehalten. $)^{46}$

Um dieses kulturelle Phantasma zu bedienen, bedarf es dieses spezifischen Helden dabei gar nicht. Dies liegt zum einen in der Struktur des Phantasmas begründet. Blick und Name orientieren sich zwar an der Figur des Despoten, sind eigentlich aber von ihr unabhängig, denn ihre Allpräsenz und Allmacht wird von den Untertanen verinnerlicht. Insofern könnte die Position des Despoten genauso gut auch leer bleiben, ${ }^{47}$ und die Melodramen um Haroun Al-Rashid spielen mit dieser Möglichkeit, indem sie zum Beispiel immer wieder eine vermeintliche Unsichtbarkeit des Kalifen dramatisieren. Damit finden sich in den Melodramen nicht nur ganz typische prämoderne Märchenmotive und -strukturen, sondern auch ein Bezug zu modernen Gesellschaftstechniken: Jeremy Bentham hatte Ende des 18. Jahrhunderts mit seinem ,Panoptikum' einen ganz ähnlichen Mechanismus der Überwachung imaginiert, der für Michel Foucault in der modernen Überwachungsgesellschaft resultiert. ${ }^{48} \mathrm{Zum}$ anderen lässt sich das kulturelle Phantasma auch auf andere Figuren übertragen. In Milners Barmecide ist dies zum Beispiel zunächst der Wesir Giafar. In letzter Konsequenz kann es auch das Publikum sein, dem ein omnipräsenter Blick zugedacht wird, der als einziger immer schon alles gesehen hat. Ein solcher panoptischer Herrscherblick des Publikums trägt dann auch eine koloniale Komponente - im Sinne eines gemeinschaftlichen Einübens machtvoll-aneignender Blicke, welches seine Entsprechung auch in anderen Kulturtechniken, etwa dem orientalistischen Panorama, findet. ${ }^{49}$

Das Phantasma des Despoten als funktionale Leerstelle lässt sich noch in einem weiteren Sinn produktiv auf Haroun anwenden: Es mag helfen, die Inszenierungen Harouns historisch, auf der Schwelle zur Moderne, näher zu bestimmen. Anhand

45 Vgl. Gertrude Himmelfarb: The Idea of Poverty. England in the Early Industrial Age. London und Boston 1984.

46 Gedruckt als: Thomas Dibdin: Haroun Alrashid; or, Wants and Superfluities. A Melodramatic Romance in Three Acts. London [o. J.].

47 Laut Grosrichard internalisieren Untertanen die Allmacht des Herrschers; ihr Begehren und die Aussicht auf Genuss treiben die Unterwerfungsmaschinerie an; vgl. Grosrichard: The Sultan's Court (Anm. 38), 164-165.

48 Für Foucault stellt das Panoptikum eine strukturelle Form der Machtausübung dar, bei der überwachende Blicke verinnerlicht werden und zu Selbstdisziplinierungen führen; vgl. Michel Foucault: Überwachen und Strafen. Die Geburt des Gefängnisses. Frankfurt am Main 101992.

49 Vgl. Edward Ziter: The Orient on the Victorian Stage. Cambridge [u.a.] 2003, Kap. 1, sowie Mary Louise Pratt: Imperial Eyes. Travel Writing and Transculturation. London und New York 1992. 
von William Dimonds The Athiop; or, The Child of the Desert, meinem letzten Fallbeispiel, lässt sich gut demonstrieren, wie die Inszenierungen Harouns zwischen vormodernen und modernen Repräsentationsformen changieren, die ihrerseits wiederum eng an die jeweilige Aufführungssituation gebunden sind. The Athiop wurde 1812 am Covent Garden Theatre uraufgeführt und handelt von einer zunächst auch erfolgreichen Revolution gegen den Kalifen. Wenn in der ersten Szene revolutionäre Handzettel (bills) ausgehängt werden, so hat dies 1812 eine aktuelle gesellschaftliche Relevanz: Es gab starke, ganz unterschiedlich motivierte radikale Strömungen in der Londoner Gesellschaft, denen wiederum durch eine zunehmend restriktive Polizeigesetzgebung begegnet wurde. ${ }^{50}$ Auch Harouns panoptische Blickmacht findet ihre zeitgenössische Entsprechung in einem omnipräsenten Blick der Obrigkeit. Öffentliche Versammlungsorte wie Kneipen, Klubs und Theater wurden zunehmend systematisch von einem Netzwerk von Informanten und Spionen des Home Office überwacht: „Britain 1790-1820 was a spy culture“, so David Worrall. (Sein Studium der Akten des Home Office hat übrigens ergeben, dass zu den prominenten Opfern dieser ,panoptischen “ Überwachung auch Jeremy Bentham selbst zählte.) $)^{51}$

Die Revolution gegen Haroun, die das Melodrama inszeniert, hat somit auch für England eine gewisse gesellschaftspolitische Sprengkraft. Dies gilt umso mehr, als sie in ein recht konventionelles Heldennarrativ gegossen wird und als legitimer Kampf eines jungen Prinzen gegen den vermeintlichen „Tyrannen“ Haroun AlRashid ausgestaltet wird. ${ }^{52}$ Zudem wird dieses Heldennarrativ (in Teilen fast exzessiv) christlich überformt - der Prinz ist eine kaum verstellte Christusfigur, seine Unterstützer werden als verfolgte urchristliche Gemeinde repräsentiert, die in Katakomben ihre gefallenen Helden als Märtyrer verehrt. ${ }^{53}$ Wenn muslimische Figuren

50 Der Diener des Dichters Percy Bysshe Shelley wurde zum Beispiel im gleichen Jahr, 1812, wegen exakt dieses Vergehens, bill-sticking, öffentlichkeitswirksam zu sechs Monaten Haft verurteilt. David Worrall hat diese „radikale Kultur“ und die Reaktionen der Obrigkeit anhand von Akten des Home Office rekonstruiert; vgl. David Worrall: Radical Culture. Discourse, Resistance and Surveillance, 1790-1820. London 1992; zum bill sticking vgl. insbesondere 44-46, zu Shelleys Diener vgl. 5. Meinungsfreiheit ist auch in anderen Melodramen um Haroun Al-Rashid ein Thema - teilweise mit unmittelbarem lokalen Bezug. So wurde Milners Barmecide 1818 am Drury Lane Theatre aufgeführt, das genau wie Covent Garden in dem Bereich lag, den das Seditious Meetings Act ein Jahr zuvor zur Bannmeile für Versammlungen deklariert hatte.

51 Worrall: Radical Culture (Anm. 50), 7; vgl. 96.

52 William Dimond: The Athiop; or, The Child of the Desert: A Romantic Play, in Three Acts. New York 1813, 31. Die Legitimierung erfolgt dabei über die Generation der Väter; Harouns Vater stürzte den Vater des Prinzen in einer blutigen Revolution vom Thron - nicht Haroun selbst, wie Bridget Orr argumentiert (Bridget Orr: Galland, Georgian Theatre, and the Creation of Popular Orientalism. In: Saree Makdisi und Felicity Nussbaum [Hg.]: The Arabian Nights in Historical Context: Between East and West. Oxford 2008, 103-129, hier 116).

53 Das Drama greift damit bereits früh einen Mythos über urchristliche Verfolgung auf, der sich bereits in populären christlichen Heldengeschichten Anfang des 19. Jahrhunderts fand (so in Alban Butlers immer wieder aufgelegtem The Lives of the Fathers, Martyrs and Other Principal Saints [London 1756-1759]). Von den 1840er Jahren an wird dieser Mythos in 
im frühen Melodrama christlich überformt werden, garantiert dies üblicherweise die Rechtmäßigkeit ihres Anliegens, wenn nicht gar den Erfolg ihres Kampfes. In diesem Melodrama aber hilft es lediglich, eine sehr traditionelle Heils- und Heldengeschichte symbolisch und narrativ auszugestalten - dann jedoch scheitert der Prinz in seinem Kampf. Mehr noch: In der letzten Szene wird die christliche Heldengeschichte fast komplett desavouiert und ihre Rhetorik als PR entlarvt, gesteuert vom verkleideten Haroun selbst. In dieser Szene haben die Revolutionäre bereits den Dolch über den vermeintlich schlafenden Kalif erhoben. In diesem Moment öffnet sich schlagartig (,the entire grouping and illumination is instant and simultaneous" $)^{54}$ die Szenerie links, rechts und oberhalb der Bühnenmitte. Diese theatrale Neurahmung entspricht einem vollständigen Perspektivwechsel; die Machtverhältnisse verkehren sich ins Gegenteil, und es stellt sich heraus, dass Haroun die gegen ihn gerichtete Revolution immer schon umfasst hatte. Er selbst war, verkleidet als „Aethiop“, ihr Motor - und zwar als Schauspieler in blackface: der fremdkulturelle Held hatte die ersten drei Akte einen fremderkulturellen Agitator gegeben. ${ }^{55}$

Diese Re-Inszenierung der Herrscheridentität muss dabei sehr prononciert erfolgen. Über das Motiv des Herrschers in Verkleidung wird nämlich nicht nur demonstriert, wie ein Herrscher auch transgressive Identitäten in seiner Person zu assimilieren vermag. Die andere Seite der Medaille ist, dass seine Herrscheridentität durch transgressive Identitäten immer auch infrage gestellt wird. Bridget Orr sieht Al-Rashids Verkleidungen daher als systematischen Versuch, einer grundsätzlichen Transgressivität gesellschaftlicher Identitäten ,Herr ${ }^{`}$ zu werden: „To expel what appears to be systemic transgression, the very source of authority incorporates transgressive identity and, however happy the outcome, Alraschid's habitual recourse to this strategy suggests the most intimate imbrication of proper authority and its negative ". ${ }^{56}$ Dies gilt umso mehr für Melodramen, in denen sich Figuren gerade auch situativ und relational erschließen, und im besonderen Maße für solche meta-

den unterschiedlichsten Bereichen popularisiert werden - von der Archäologie über die Religionswissenschaften bis hin zur touristischen Reiseliteratur zu den Katakomben Roms, so zum Beispiel Selina Bunbury: A Visit to the Catacombs, or First Christian Cemeteries at Rome. London 1849.

54 Dimond: The Æthiop (Anm. 52), 72.

55 Vgl. Orr: Galland (Anm. 52), 116.

56 Ebd., 115. Eine solche Metatheatralität zeichnet nicht nur die Dramen um Haroun AlRashid aus. Katherine Newey hat argumentiert, dass Melodramen, insbesondere im späteren 19. Jahrhundert, sehr viel systematischer metatheatralische Elemente inkorporieren, die auch in politischen Kontexten von Heroisierungen stehen: „The political nature of metatheatricality is foregrounded in melodrama. The heightened world of melodrama [...] is a defence of the heroic feeling individual against the encroachments of industrialisation and urbanisation. The principle of metatheatricality is central here, as such a theatrical defence of the heroic might otherwise be undermined by Victorian anti-theatrical suspicion of the fluidity and malleability of the theatrical character." (Katherine Newey: Melodrama and Metatheatre: Theatricality in the Nineteenth Century Theatre. In: Journal of Dramatic Theory and Criticism 11: 2, 1997, 85-100, hier 98-99) 
theatralen Momente, bei denen die Theatralität von Herrschaft selbst zum Thema zu werden droht.

Insofern ist diese letzte Szene des Melodramas durchaus typisch: Haroun inszeniert seine Macht durchweg als ein Prinzip, das vermeintlich immer schon funktioniert hat. Die agonistischen Elemente, welche die Dramen auffahren (eine Revolution, ein verstoßener Erzfeind, eine Bedrohung durch antisoziale Figuren aus der Wüste), sind, wie The Ethiop ausbuchstabiert, eigentlich schon immer besiegt gewesen; es bedurfte nur einer leicht verschobenen Perspektive, um dies zu erkennen. Am Ende des Dramas hat Haroun den orientalischen Ersatzchristus schlicht in sein Machtgefüge integriert - als seinen Adoptivsohn. ${ }^{57}$ Insofern hat sich Haroun den Heiland des ersten Heldennarrativs angeeignet und ihn in eine neue, übergeordnete Heilsgeschichte inkorporiert. Das Melodrama legt solche prämodernen Deutungsmuster durchaus nahe, insbesondere auch in Verbindung mit seinen märchenhaften Motiven und Strukturen. Gleichzeitig weist das Mechanische, Apparaturhafte von Harouns Macht aber auch auf moderne, entpersonalisierte Herrschaftstechniken.

In der Möglichkeit dieses Nebeneinanders deutet sich für mich der Kern der Inszenierungen Harouns an. Haroun ist vielleicht auch deshalb an der Schwelle zur Moderne ein so populärer Held, weil er, trotz aller Personalisierungen (etwa als „Vater des Volkes"), trotz seiner Abenteuer gegen Revolutionäre, eine Leerstelle beziehungsweise ein bloßes Prinzip repräsentiert. Die auratische Aufladung seiner Person in spektakulären Szenen will dies gar nicht überdecken, sie fetischisiert es vielmehr. In diese Leerstelle ist nun aber Alterität machtvoll einschreibbar - und zwar gleichzeitig prämodern (märchenhaft-magisch, paternalistisch-feudalistisch, heilsgeschichtlich) und modern: Innerhalb einer modernen Gesellschaftstheorie kann der Herrscherblick bereits als ubiquitärer Blick der Überwachung fungieren. Und in einer zunehmend geordneten und durchregulierten bürgerlichen Gesellschaft sind Harouns Verkleidungen als Verbrecher oder als Räuber vielleicht die (intra-kulturell) letzten , anderen' Helden. ${ }^{58}$

Ich hatte damit begonnen herauszustellen, dass Melodramen in vielerlei Hinsicht selbst als ein ,Anderes' fungieren. Aufgrund ihrer, Anders'-Artigkeit dienen sie ihrer Zeit aber auch als ein kulturelles Experimentierfeld: Sie integrieren Affekthaftes als einen zentralen ästhetischen Bestandteil, und in einer Zeit, in der ,eigene' Herrscher (klassische Nationalherrscher) als Theaterhelden weitgehend ausge-

57 Prinz Orasmyn ist auch für Melodramenverhältnisse ein (im Wortsinne) schwacher Protagonist, der aufgrund von Schwäche und Ohnmachtsanfällen beständig gestützt werden muss. Er übernimmt bis zu einem gewissen Grad eine der konventionalisierten Rollen einer melodramatischen Protagonistin, wie sie Merle Tönnies beschrieben hat; vgl. Merle Tönnies: (En-)Gendering a Popular Theatrical Genre. The Roles of Women in Nineteenth-Century British Melodrama (Anglistische Forschungen; 443). Heidelberg 2014, 75-102.

58 Vgl. Burwick: Playing to the Crowd (Anm. 14), Kap. 7 zu „Heroic Rebels and Highwaymen“ auf der Bühne. Ich verdanke diese Lesart Doris Feldmann. 
dient haben, entwerfen Melodramen einen in die (konventionalisierte) ,Fremde verschobenen, imaginären Ersatz. Und schließlich spielen Melodramen andere Möglichkeiten durch, wie ein heroischer Überschuss, eine Exzeptionalität des Helden, ausgestaltet werden kann - nicht primär vertikal (etwa sozial oder staatlich), sondern vor allem horizontal, dafür aber in immer breiteren Formen: Der bürgerliche Held im domestic melodrama oder der Arbeiterheld im industrial melodrama wird nun moralisch, emotional oder imaginär nobilitiert. Der melodramatische Held wird in der Folge ,einfach' anders. 